\author{
Military Technical College \\ Kobry El-Kobbah, \\ Cairo, Egypt.
}

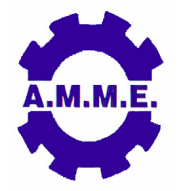

\title{
SCORING MODEL FOR FIBER LASER CUTTING OF MILD STEEL SHEETS
}

$15^{\text {th }}$ International Conference
on Applied Mechanics and
Mechanical Engineering.

M. Sobih ${ }^{*}$ and W.M. Hussien ${ }^{*}$

\begin{abstract}
Laser cutting is one of the most important non-conventional manufacturing processes due to its unique properties and advantages. It is a multi-parameter technology. This fact has resulted in some gaps in available information, and a few of contradictory reports about the exact effect of each process parameter on the surfaces produced, and makes quick parameters selection for obtaining a desired cut quality problematic, especially for new laser types. Fiber lasers have only very recently come into prominence, and the literature is still very sparsely populated with data on their performance. This paper outlines the use of a scoring model of Projection to Latent Structure (PLS) technique and applies it to study the effects of fiber laser cutting process parameters and their interactions on the quality achieved during cutting mild steel sheets. The effects of the interaction of the main process parameters on the quality output variables such as top kerf width, surface roughness and the striation depth are studied. Scoring models are proposed to relate the quality and the laser cutting parameters.
\end{abstract}

\section{KEY WORDS}

Laser cutting; mild steel cutting; cutting parameters; cutting quality; multivariate analysis; and Projection to Latent Structure.

Egyptian Armed Forces. 


\section{INTRODUCTION}

Laser cutting is one of the most common and best established industrial laser applications. The process is characterized by number parameters affecting the process individually or in combination. These parameters can be divided to three categories: the laser beam parameters, viz. laser power, spot size, focal position, depth of focus, and laser scanning speed (cutting speed); the material parameters, viz. material type, material thickness and its optical and thermal properties; and finally the assist gas properties, viz. gas type, pressure, nozzle exit diameter and distance between nozzle and workpiece (stand-off distance) [1].

Depending on the application the quality characteristics of the cut surface can be one or more of the following: surface roughness; surface perpendicularity (angularity); kerf width; dross adhesion; the dimensions of the heat affected zone; and the striation characteristics (wavelength, frequency, and depth).

Various studies of these parameters and quality characteristics have been reported. Early work by Gonsalves and Duley in 1970s investigated the interdependence of the laser parameters. They showed that the kerf width decreases with increasing cutting speed and workpiece thickness, while it increases with the increase of laser power. They also showed the increase of maximum cutting speed with the laser power, and a decrease with the workpiece thickness [2]. In a subsequent paper they showed that using oxygen as an assist gas increases the maximum (critical) cutting speed due to the exothermic chemical reaction in the cutting zone. Also the cutting speed increases with an increase in oxygen flow rate and again at a very high oxygen flow rate decreases because of the cooling of the cutting zone due to the heat transfer to the impinging gas, [3]. These were supported by others [4, 5], while the results of Karatas et al. [6] and Sobih et al. [7] contradict the Gonsalves and Duley [2] findings concerning the effect of workpiece thickness upon the kerf width. They showed that the kerf width increases as the workpiece thickness increases.

The effect of focal plane position upon the kerf width and the effect of the workpiece thickness upon the striation width and depth have been investigated by Karatas et al. [6]. It was found that the minimum kerf width can be achieved when using a focused beam, especially for thin workpieces, while for thick workpieces the focal plane should be moved into the work piece to minimize the kerf width. The striation width and depth were found to increase as the workpiece thickness increases.

Continuous wave (CW) $\mathrm{CO}_{2}$ laser cutting of mild and stainless steels (both exothermic and fusion) were investigated by Hamoudi [4]. He demonstrated that the cut quality improves and the heat affected zone (HAZ) decreases as the cutting speed increases and the assist gas pressure decreases. He also found exothermic laser cutting of stainless steel to be better than fusion laser cutting.

All the foregoing studies followed a one-factor-at-a time as strategy for studying and analyzing the laser cutting process. Contradictions are therefore to be expected. More comprehensive studies include that of Tam et al., [8], who studied the laser cutting of low carbon steel with respect to a figure-of-merit function using a modified L8 orthogonal array. They concluded that the use of Taguchi's parameter design and ANOVA enables the drawing of qualitative and objective conclusions. Rajaram et al. 
[9] developed a regression model to predict the effect of laser power and cutting speed on the kerf width, surface roughness and heat affected zone when cutting 4130 steel using a $\mathrm{CO}_{2}$ laser. Wee et al. [10] introduced a statistical model based on the multivariate regression to determine the parameters affecting the cut quality when cutting ceramic with laser. They found that interaction time has a major effect on both striation angle and wavelength, while irradiance is significant factor in striation wavelength.

Multivariate statistical process control (MSPC) takes a different approach as compared to the other methods mentioned previously [11]. The most fundamental difference with this approach is that the model is based on non-causal empirical correlations extracted from normal plant operating data when only common cause variation exists [12]. The simplicity of this approach is that there is no need for a fundamental model of the system and only data from normal production needs to be used, which is generally available in some form at most factories. Two of the approaches used in multivariate analysis are: a projection method called principal component analysis (PCA) [13, 14] and projection to latent structure (PLS) [15]. Many applications of these two techniques have been successfully applied in other fields of process monitoring ranging from batch to continuous processes [16-18].

$\mathrm{CW} \mathrm{CO}$ has been the most commonly used laser for metal cutting over the last two decades and more. A recent a newcomer to the industry is the fiber laser. The advantages of fiber laser over the $\mathrm{CO}_{2}$ laser include: a small physical size; high stability of power output; high brightness; good beam quality; a narrow focus; significantly higher cutting speeds; smaller kerf widths; and higher beam absorption in metals due to its shorter wavelength [19,20]. A number of studies have been reported during the last few years either to investigate fiber laser cutting or to compare between it and other types of lasers [21,22].

This paper is an experimental study into the effect of the cutting parameters on the quality characteristics obtained when cutting mild steel using a single mode fiber laser. Seven factors were considered at different levels. The quality parameters taken include: top kerf width; surface roughness (Ra); and striation depth. A statistical study was carried out to find the most effective cutting parameters and their interactions. The scoring models correlate between the quality parameters and the significant input parameters. The work is part of a broader study that has indicated the possibility of optimizing surface roughness to the extent of obtaining essentially striation-free cut surfaces [23-25].

\section{EXPERIMENTAL}

The experiments were performed on as-received 1, 1.5 and $2 \mathrm{~mm}$ thickness sheets of EN43 annealed mild steel and were conducted using a CW IPG YLR-1000-SM ytterbium-doped single-mode fiber laser with the following specifications: $1 \mathrm{~kW}$ maximum output power; $1.07 \mu \mathrm{m}$ wavelength; $14 \mu \mathrm{m}$ output fiber core diameter; $\mathrm{M}^{2}=$ 1.1; and mode TEMo0. This machine is equipped with Precitec HP1.5"(Z)/FL cutting head with capacitive distance control. The laser beam was focused using a 5 inch $(127 \mathrm{~mm})$ focal length lens. Oxygen was used as assist gas. 
Preliminary experiments were carried out to select appropriate and achievable levels of the factors under study. These experiments showed that when the laser beam was focused on or below the work piece surface significant dross formation was observed. A focal plane set to be above the workpiece achieved superior results. The laser output power, workpiece thickness, oxygen gas pressure, stand off distance, cutting speed, nozzle diameter and laser beam spot size were the parameters investigated. They were varied during the experiments within the limits indicated in Table 1. Top kerf width was examined using optical microscopy, while surface roughness and striation depth were inspected using a laser surface profile scanning system which was developed in-house.

\section{EXPERIMENTAL DESIGN AND SCORING MODELS}

\section{Experimental Design}

Studying the dependent factors following the one-factor-at-a-time approach results in large number of experiments, and fails to consider possible interactions between these factors. A statistical design of experiments (using the software package Design-Expert) was thus preferred, based on the parameters discussed above. The design is a subset of all possible combinations of the factors and aims to minimize the error associated with the model coefficients. This design contains 114 experimental points for estimating the model using the Design of Experiments, instead of 4374 total number of possible points if one-factor-at-a time approached is used.

The method used for obtaining the experimental design was the D-Optimal Algorithm. A D-optimal design minimizes the determinant of the $\left(X^{\prime} X\right)-1$ matrix. This minimizes the volume of the confidence ellipsoid for the coefficients. Equivalently Doptimality maximizes the determinant $\left(X^{\prime} X\right)$, called the "information" matrix. The key is that these designs are built algorithmically to provide the most accurate estimates of the model coefficients, [26].

The CONVERT algorithm was used to find vertices. It is believed that this algorithm finds all vertices of the simplex, but in higher dimensions with complex constraints, it is possible that an extra point or two may be in the vertex list, [26-28].

The algorithm used to find an approximately D-optimal design is as follows: selection of a non-singular initial design of $p$ points; selection of the remaining model points; performing exchange steps. If the candidate list is large and/or the degree of the design is large, D-optimal point selection can be a lengthy process.

\section{Multivariate PLS Basic Theory}

PLS is defined as a generalized multiple regression method based on relating two blocks of data made up of input and output variables. This method is particularly effective at modeling and analyzing variables that are correlated; which is the case for many process data. 
In this case, the co-linearity within variables were tested by checking the loading plot, a scatter plot between the model weights in the first 2 principal components as shown in Figure 1. It shows that a positive correlation exists between factors 1, 7, 6, 3 and 2 in the direction of the first principal components (x-axis). Also, it shows a negative correlation between the mentioned factors 5 and 4 . An overview about the method and its applications can be found in [29].This commonly occurring colinearity between variables means that the data will have some dominant type of variability that carries most of the information. To visualize these hidden dominant directions, variables are projected onto a new subspace. This implies the reduction of a large data set from many variables to a few factor scores $(t, u)$ and loadings $(w, p, q)$. The point behind using 2 loadings $(w, p)$ to describe $x$-variables, is that in PLS algorithm, the y's is allowed to intervene directly during the input variables decomposition which decreases the variance explanation of the input variables towards the maximum predictability of the response variable. In this case we use two loadings to describe the input variables, the w's to calculate principal components and the p's when calculating the residuals for each component because it better describes input variables. Scores can be described as new generalized variables while loadings build the connection between the original space and the new subspace. The approach works by selecting factors of input variables in a sequence which successively maximizes the explained covariance between the input and output variables. Given a matrix of input data $X$, and output data, $Y$, a factor of the input data, $t_{1}$, and output data, $u_{1}$, is evaluated such that:

$$
X=\sum_{k=1} t_{k} \times p_{k}^{T}+E
$$

and

$$
Y=\sum_{k=1} u_{k} \times q_{k}^{T}+F
$$

These equations are referred to as the outer relationships where the " $\mathrm{t}$ " vectors are mutually orthogonal. These vectors together with the $u_{k}$ terms are selected so as to maximize the covariance between each pair, $\left(t_{k}, u_{k}\right)$. Linear regression is performed between the $t_{k}$ and the $u_{k}$ terms to produce the inner relationship, such that $u_{k}=b_{k} \times t_{k}+\varepsilon_{k}$, where $\mathrm{b}_{\mathrm{k}}$ is a regression coefficient, and $\varepsilon_{\mathrm{k}}$ refers to the prediction error. The PLS method provides the potential for a regularized model through selecting an appropriate number of latent variables, $u_{k}$ in the model. The major benefit of this approach is that it builds a model between the inputs as well as the outputs and finds the maximum correlation between them in the direction of maximum prediction of the output response. Furthermore, it is often found that a relatively small number of low-index latent variables can explain the greater part of the variation in both the input and output variables. Table 1 illustrates the configuration of the X-Y matrices in the models used in this paper. Where Pow is the laser power, Thick is the sheet thickness, GPre is the gas pressure, stDis is the stand off distance, speed is the cutting speed, NDia is the nozzle diameter, SpS is the laser spot size, TKW is the top kerf width, Ra is the surface roughness and StrHei is the striation height. 


\section{Scoring Models and Data Analysis}

Before starting the discussion we need to know some aspects of the approach. First, all data were mean centered and scaled to unit variance. Second, control limits in the latent space were established using F-distribution based on reference distribution provided by the dataset, [29]. Also, $\mathrm{t} 1$ and $\mathrm{t} 2$ are the first two principal components that capture most of the variance-covariance between the $X-Y$ relationships. The multivariate PLS model is a linear model given in matrix form by: $Y=X B+E$.

To build the model using process variables, let $X$ include process variables (Pow, Thick, GPre, stDis, speed, NDia, SpS), these variables were believed to have a direct correlation with the response variables (TKW, Ra, StrHei). Then let $Y$ consist of the machining response variables which are the surface roughness $\mathrm{Ra}$, TKW, and StrHei values of the machined parts. Finally, the PLS regression is made between $X$ and $Y$ based on an algorithm which is superficially "similar" to NIPALS (Nonlinear Iterative Partial Least Squares) algorithm [13]. All the models were built using Matlab-7 and SIMCA-P code developed by Umetrics. To examine the relationship between the inputs and the output in the latent space, Figure 2 shows a scatter plot between the experimental and predicted variables. It illustrates a linear trend between them which imply the possibility to use the approach for further analysis.

An exploring model was built based on PLS to examine the relationship between the controlled factors (Pow, Thick, GPre, stDis, speed, NDia, SpS) and the three response variables (TKW, $\mathrm{Ra}$, StrHei) using two principal components with the following percentage explanation: $R^{2} X$ (cum): $0.46, R^{2} Y$ (cum): 0.51, $Q^{2}$ (cum): 0.4.

Figure 3 and Figure 4 illustrate the loading and score plots of the model. The score plot illustrates the effect of using D-optimal in designing the experiments with the good data spread inside the control limits. The loading plot shows how all the variables are related to each other. As mentioned above, one can notice the high influence of Sps and Pow on tkw. Also, the effect of Speed, NDia, GPre and thick on $\mathrm{Ra}$ and StrHei. In addition, the plot shows the poor effect of stDis on all the three responses.

Detailed models are set to examine the effect of process variables on each response parameters. A first model was built based on PLS to examine the relationship between the controlled factors (Pow, Thick, GPre, stDis, speed, NDia, SpS) and the response variable TKW using two principal components with the following percentage explanation: $R^{2} X$ (cum): $0.4, R^{2} Y$ (cum): $0.665, Q^{2}$ (cum): 0.53 .

Figure 5 illustrates the loading plot of the model. The loading plot shows how all the variables are related to each other. In this analysis, one can observe the positive correlation of Sps and Pow on tkw with the poor negative effect of the Speed.

Figure 6 and Figure 7 summarize these effects showing variable importance and positive and negative effects on the response variable "TKW".

The PLS deduced model of TKW with x-parameters:

$$
\mathrm{Y}(\mathrm{TKW})=0.4959 \text { Pow }-0.1658 \text { Thick }-0.2367 \mathrm{Spd}+0.6181 \mathrm{Sps}
$$


A Second model was built based on PLS to examine the relationship between the controlled factors and the response variable surface roughness (Ra) using two principal components with the following percentage explanation: $R^{2} X$ (cum): 0.31 , $R^{2} Y$ (cum): 0.35, $Q^{2}$ (cum): 0.18 .

Figure 8 illustrates the loading plot of the model. In this analysis, one can observe the positive correlation of GPre and Thick on Ra with the good negative effect of the Speed.

Figure 9 summarizes these effects showing variable importance effects on the response variable Ra. The PLS deduced model of Ra with x-parameters:

$$
\begin{array}{rrlll}
\mathrm{Y}(\mathrm{Ra}) & =0.2042 & \text { Pow }+0.1024 & \text { Thick }+0.3083 \text { GPre } \\
& -0.1282 & \text { StDis }-0.4573 & \text { Spd }+0.1578 \text { NDia }
\end{array}
$$

A third model was built based on PLS to examine the relationship between the controlled factors (Pow, Thick, GPre, stDis, speed, NDia, SpS) and the response variable StrHei using two principal components with the following percentage explanation: $R^{2} X$ (cum): 0.312, $R^{2} Y$ (cum): $0.535, Q^{2}$ (cum): 0.41 .

Figure 10 illustrates the loading plot of the model. In this analysis, one can notice the positive correlation of GPre and Thick on StrHei with the good negative effect of the Speed.

Figure 11 summarizes these effects showing variable importance and effects on the response variable StrHei. The PLS deduced model of StrHei with x-parameters:

$$
\begin{aligned}
\mathrm{Y}(\text { StrHei })= & 0.0346 \text { Pow }+0.1144 \text { Thick }+0.3194 \text { GPre } \\
& -0.2549 \text { StDis }-0.57 \text { Spd }+0.1840 \text { NDia }
\end{aligned}
$$

\section{CONCLUSIONS}

The results of the d-optimal statistical analysis presented here demonstrate that a reduced number of tests are useful for indicating the significant process parameters for the laser cutting process. Using this method, seven parameters relating to the fiber laser cutting of mild steel process were studied and the following conclusions were drawn: first, the top kerf width is directly related to the laser output power and laser beam spot size and inversely related to cutting speed and sheet thickness while the gas pressure, stand of distance and nozzle diameter have a minor effect; secondly, the cut surface quality is directly related to laser power, sheet thickness, gas pressure and nozzle diameter and inversely related to both the cutting speed and stand of distance while the beam spot size is not significant.

Three equations were deduced from the experimental results using scoring models of PLS technique. These equations relate top kerf width, surface roughness $\left(R_{a}\right)$ and striation height to their significant parameters. The empirical equations can be used to predict the top kerf width and the surface roughness of the cut produced in mild 
steel using fiber laser knowing the cutting conditions. This could be helpful in building up a data base for the mild steel cutting using fiber laser.

\section{REFERENCES}

[1] Ion, J.C., Laser processing of engineering materials: principles, procedures, and industrial application. 2005: Oxford: Elsevier/Butterworth-Heinemann.

[2] Gonsalves, J.N. and W.W. Duley, Cutting thin metal sheets with the CW $\mathrm{CO}_{2}$ laser. Journal of applied physics, 1972. 43(11): p. 4684-4687.

[3] Duley, W.W. and J.N. Gonsalves, $\mathrm{CO}_{2}$ laser cutting of thin metal sheets with gas jet assist. Optics and laser technology, 1974. 6(2): p. 78-81.

[4] Hamoudi, W.K., Effects of speed and processing gas on laser cutting of steel using a $2 \mathrm{~kW} \mathrm{CO}_{2}$ laser. International journal for the joining of materials, 1997. 9(1): p. 31-36.

[5] Uslan, I., $\mathrm{CO}_{2}$ laser cutting: Kerf width variation during cutting. Proceedings of the institution of mechanical engineers, Part B: Journal of engineering manufacture, 2005. 219(8): p. 571-577.

[6] Karatas, C., O. Keles, I. Uslan, and Y. Usta, Laser cutting of steel sheets: Influence of workpiece thickness and beam waist position on kerf size and striation formation. Journal of materials processing technology, 2006. 172(1): p. 22-29.

[7] Sobih, M., P.L. Crouse, and L. Li. Laser cutting of variable thickness materials-understanding the problem. in $25^{\text {th }}$ International congress on applications of lasers and electro-optics ICALEO06. 2006. Scottsdale, AZ, USA.

[8] Tam, S.C., L.E.N. Lim, and K.Y. Quek. Application of Taguchi methods in the optimization of the laser-cutting process. in 3rd Joint NTU/NUS Seminar on materials processing, Journal of materials processing technology. 1992. Singapore, Singapore.

[9] Rajaram, N., J. Sheikh, and S.H. Cheraghi, $\mathrm{CO}_{2}$ lasers cut quality of 4130 steel. International journal of machine tool and manufacture, 2003. 43: $p$. 351-358.

[10] Wee, L.M., P.L. Crouse, and L. Li, A statistical analysis of striation formation during laser cutting of ceramics. International Journal of Advanced Manufacturing Technology, 2007. DOI 10.1007/s00170-006-0888-5.

[11] Kourti, T. and J.F. MacGregor, Multivariate SPC methods for process and product monitoring. Journal of quality technology, 1996. 28(4).

[12] Yoon, S. and J.F. MacGregor, Fault diagnosis with multivariate statistical models part 1: using steady state fault signatures. Journal of process control, 2001. 11: p. 387-400.

[13] Wold, S., K. Esbensen, and P. Geladi, Principal component analysis. Chemometrics and intelligent laboratory systems, 1987. 2: p. 37-52.

[14] Jackson, J.E., A User's Guide to Principal Components. 1991: Wiley, New York.

[15] Wold, S., M. Sjostrom, and L. Eriksson, PLS-regression: a basic tool of chemometrics. Chemometric and intelligent laboratory systems, 2001. 58: p. 109-130. 
[16] Lennox, B., H.G. Hiden, G.A. Montague, G. Kornfeld, and P.R. Goulding, Application of multivariate statistical process control to batch operation. Computers and chemical engineering, 2000. 24(2): p. 291-296.

[17] Goulding, P.R., B. Lennox, D.J. Sandoz, K.J. Smith, and Marjanovic, Fault detection in continuous processes using multivariate statistical methods. International journal of systems science, 2000. 31(11): p. 1459-1471.

[18] Tsung, F., Improving Automatic-Controlled process quality using adaptive principal component monitoring. Quality Reliability Engineering Int., 1999. 15: p. 135-142.

[19] Kleine, K.F. and K.G. Watkins. Fiber laser for micro cutting of metals. in SPIE - The international society for optical engineering: Advances in fiber lasers. 2003. San Jose, CA, United States: The International Society for Optical Engineering.

[20] Liu, W., W. Du, and J. Liao. Application of fiber laser used in the field of stent cutting and micro-machining. in Lasers in material processing and manufacturing II. 2005. Beijing, China: International Society for Optical Engineering, Bellingham, WA 98227-0010, United States.

[21] Baumeister, M., K. Dickmann, and T. Hoult, Fiber laser micro-cutting of stainless steel sheets. Applied Physics A: Materials science and processing, 2006. 85(2): p. 121-124.

[22] Sparkes, M., M. Gross, S. Celotto, T. Zhang, and W. O'Neill. Inert cutting of medium section stainless steel using a $22 \mathrm{Kw}$ high brightness fiber laser. in $25^{\text {th }}$ International congress on applications of lasers and electro-optics, ICALEO06. 2006. Scottsdale, AZ, USA.

[23] Sobih, M., P.L. Crouse, and L. Li, Eliminating striation in laser cutting of mild steel. Journal of Physics D (Applied Physics), Accepted in Sep. 2007.

[24] Sobih, M., P.L. Crouse, and L. Li, Striation-free fibre laser cutting of mild steel. Applied Physics A, Accepted in July 2007. DOI: 10.1007/s00339-0074247-7.

[25] Li, L., M. Sobih, and P.L. Crouse, Striation-free Laser Cutting of Mild Steel Sheets. CIRP Annals - Manufacturing Technology, 2007. 56(1): p. 193-196.

[26] Mitchell, T.J., Algorithm for the construction of "D-Optimal" experimental deign. Technometrics, 1974. 16(2): p. 203-210.

[27] Snee, R.D., Experimental designs for mixture systems with multicomponent constraints. Communications in Statistics - Theory and Methods, 1979. A8(4): p. 303-26.

[28] Piepel, G.F., Programs for generating extreme vertices and centroids of linearly constrained experimental regions. Journal of Quality Technology, 1988. 20(2): p. 125-39.

[29] MacGregor, J.F., Using on-line process data to improve quality. ASQC, statistical division newsletter, 1995. 16(2). 
Table 1: Cutting parameters and their levels.

\begin{tabular}{l|c|c|c} 
& Level 1 & Level 2 & Level 3 \\
\hline \hline $\mathrm{x}_{1}:$ Laser power $(\mathrm{W})$ & 200 & 500 & 800 \\
$\mathrm{x}_{2}$ : Material thickness $(\mathrm{mm})$ & 1 & 1.5 & 2 \\
$\mathrm{x}_{3}$ : Gas pressure $(\mathrm{bar})$ & 2 & 6 & 10 \\
$\mathrm{x}_{4}$ : Stand of distance $(\mathrm{mm})$ & 1 & 1.5 & 2 \\
$\mathrm{x}_{5}:$ Cutting speed $(\mathrm{mm} / \mathrm{s})$ & 20 & 60 & 100 \\
$\mathrm{x}_{6}:$ Nozzle diameter $(\mathrm{mm})$ & 1 & 1.5 & 2 \\
$\mathrm{x}_{7}:$ Laser beam diameter & 0.169 & 0.336 & \\
$(\mu \mathrm{m})$ & & & \\
\hline
\end{tabular}

Table 2: Table 1; X-Y matrix.

\begin{tabular}{|c|c|c|c|c|c|c|c|c|c|}
\hline \multicolumn{4}{|c|}{ X-matrix } & \multicolumn{3}{|c|}{ PLS-model } & & & Y-matrix \\
\hline Pow & Thick & GPre & stDis & speed & NDia & SpS & TKI & & StrHei \\
\hline & & & & & & & & & \\
\hline & & & & & & & & & \\
\hline & & & & & & & & & \\
\hline
\end{tabular}




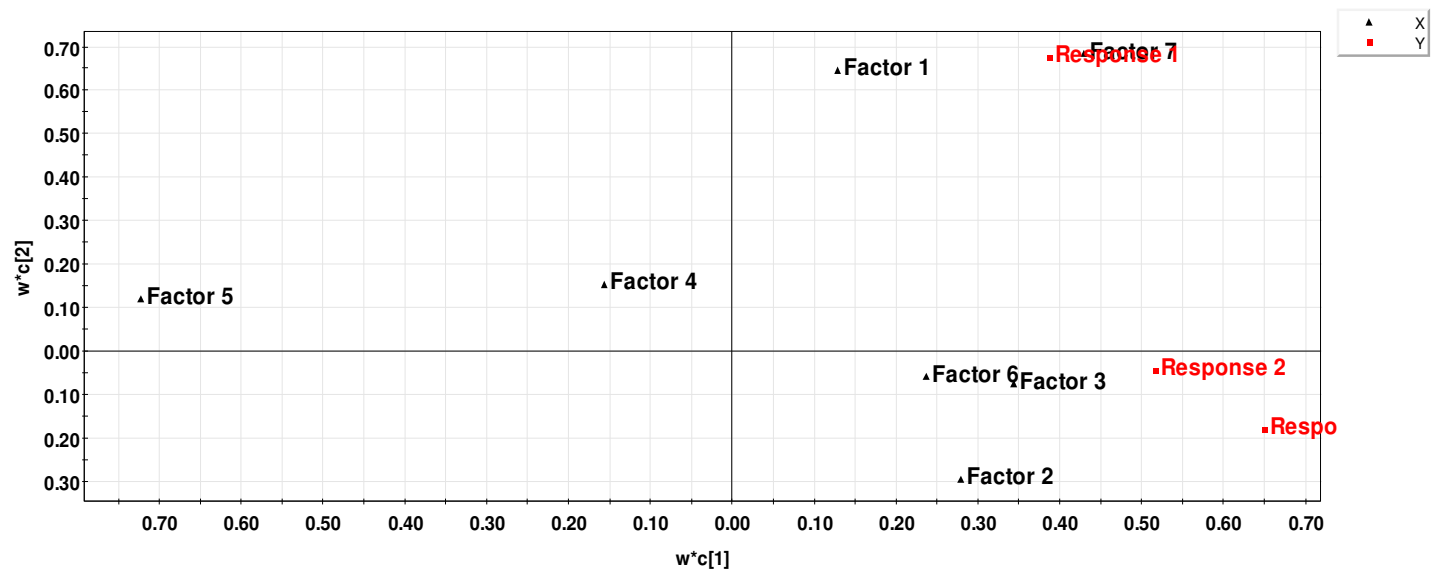

Figure 1: PLS-model loading plot.

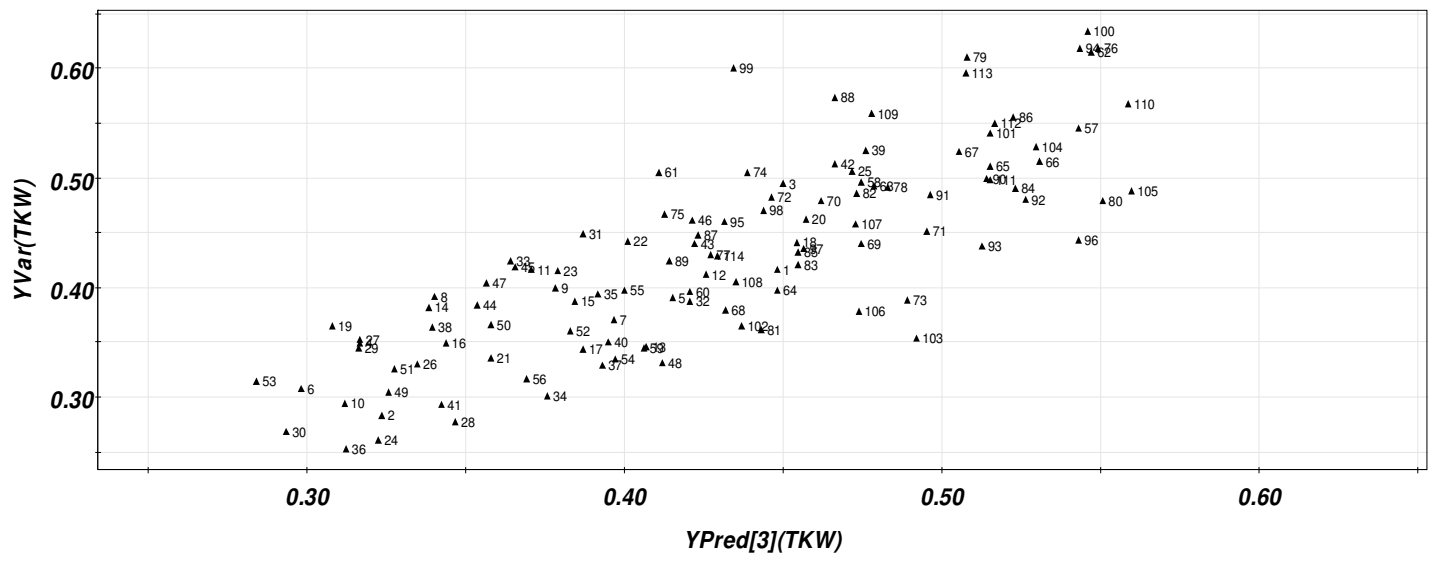

Figure 2: PLS t-u Score plot.

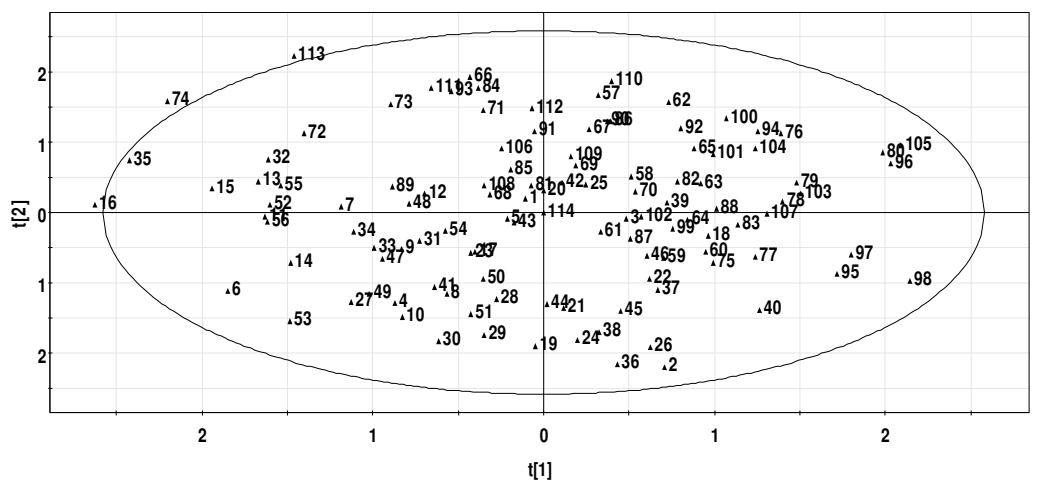

Figure 3: PLS Score plot. 


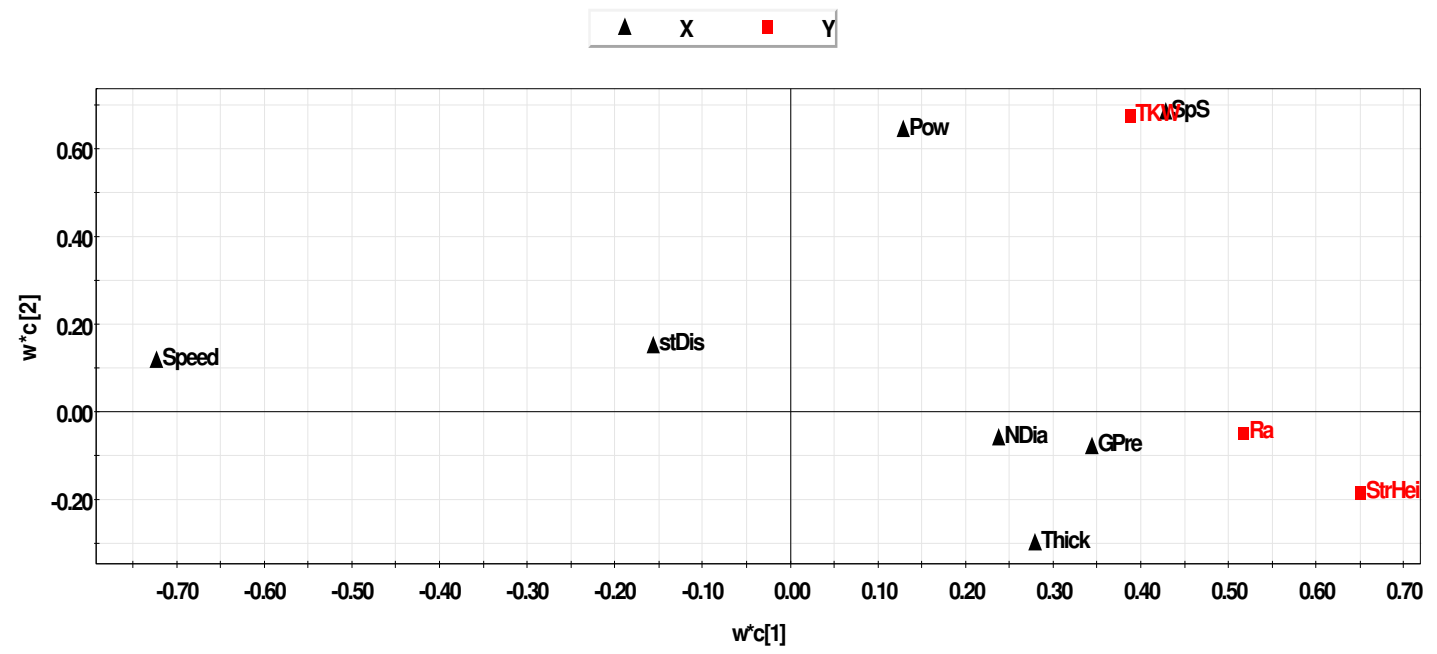

Figure 4: PLS loading plot.

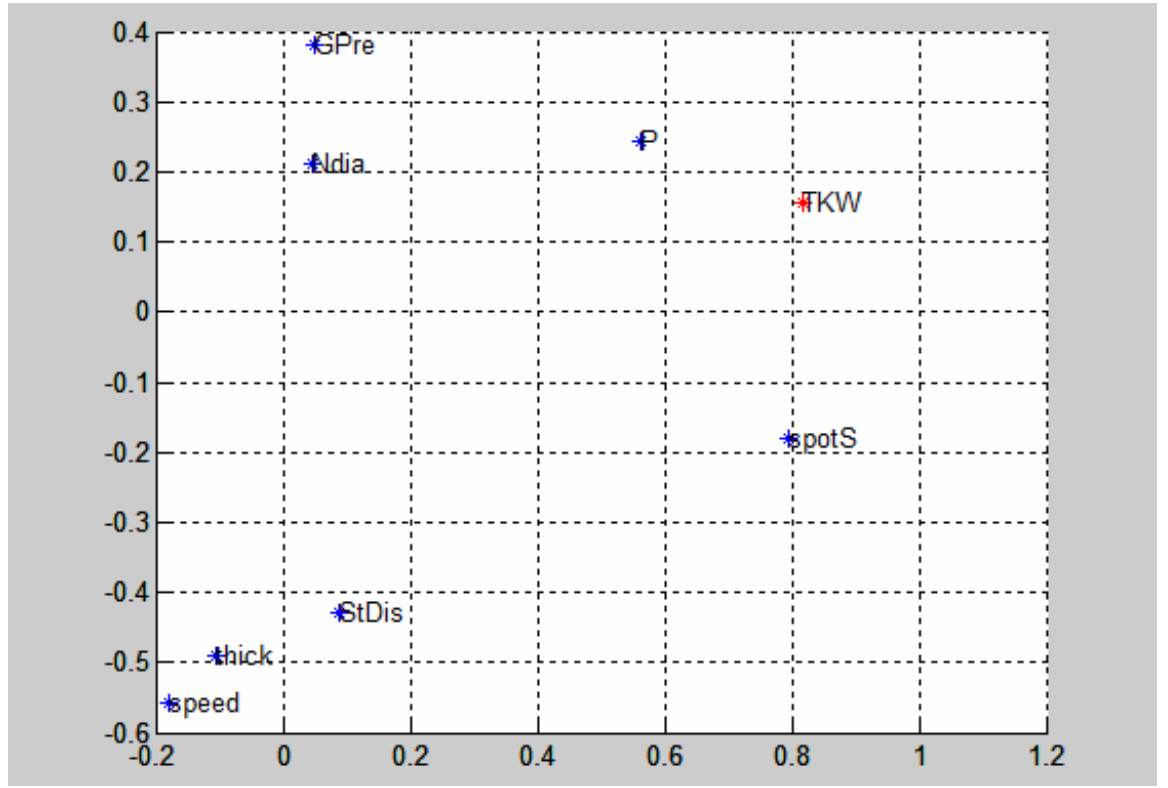

Figure 5: PLS loading plot.

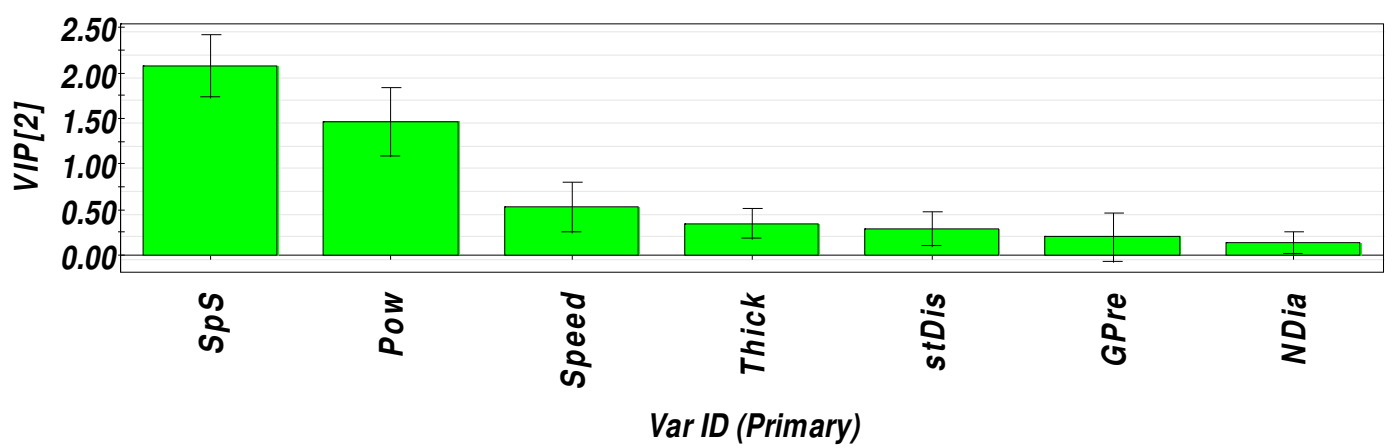

Figure 6: PLS VIP plot. 


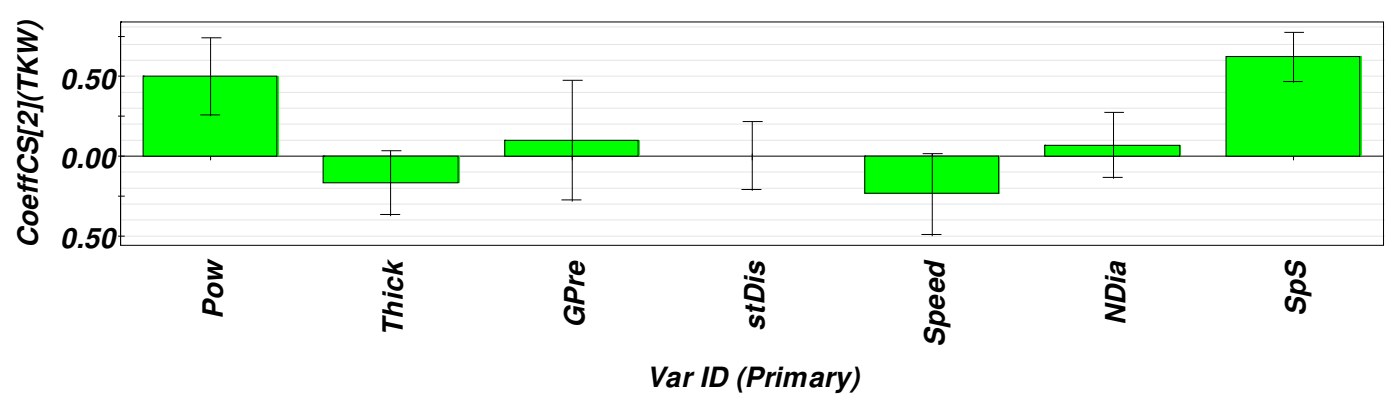

Figure 7: PLS coefficient plot.

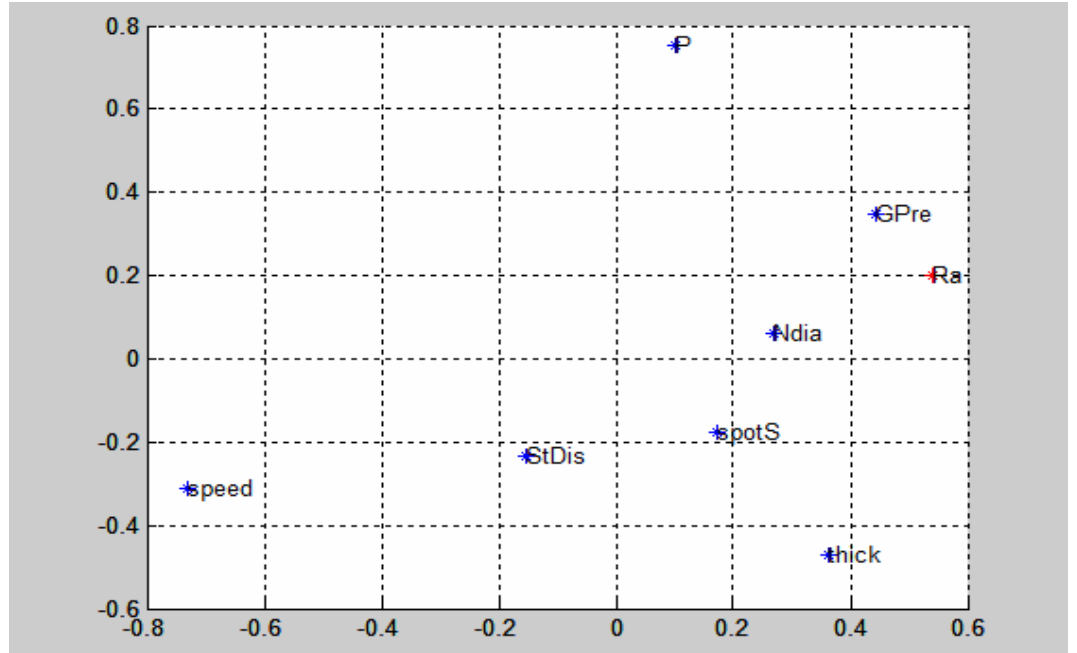

Figure 8: PLS loading plot.

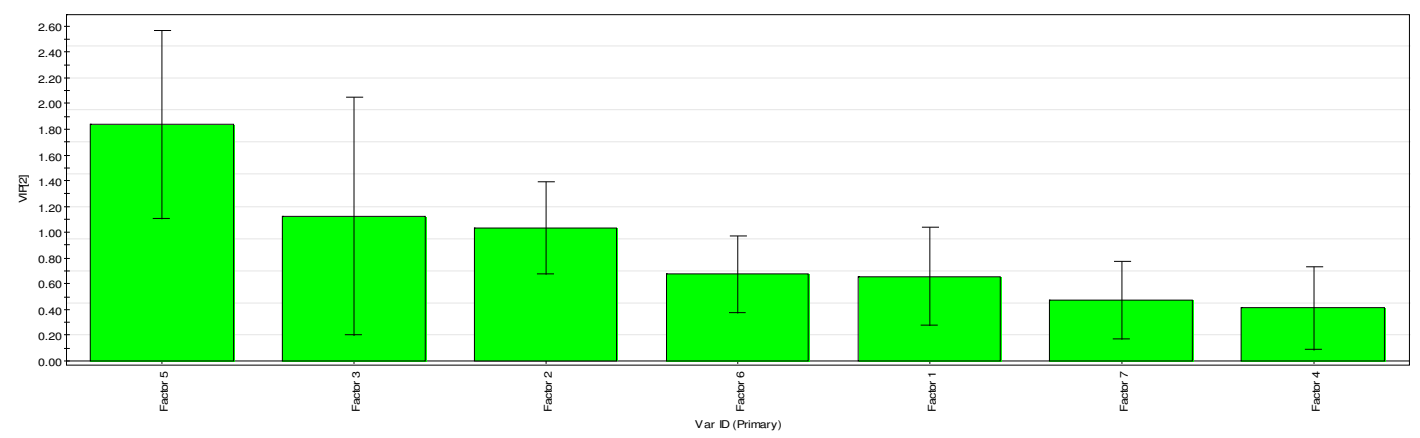

Figure 9: PLS VIP plot. 


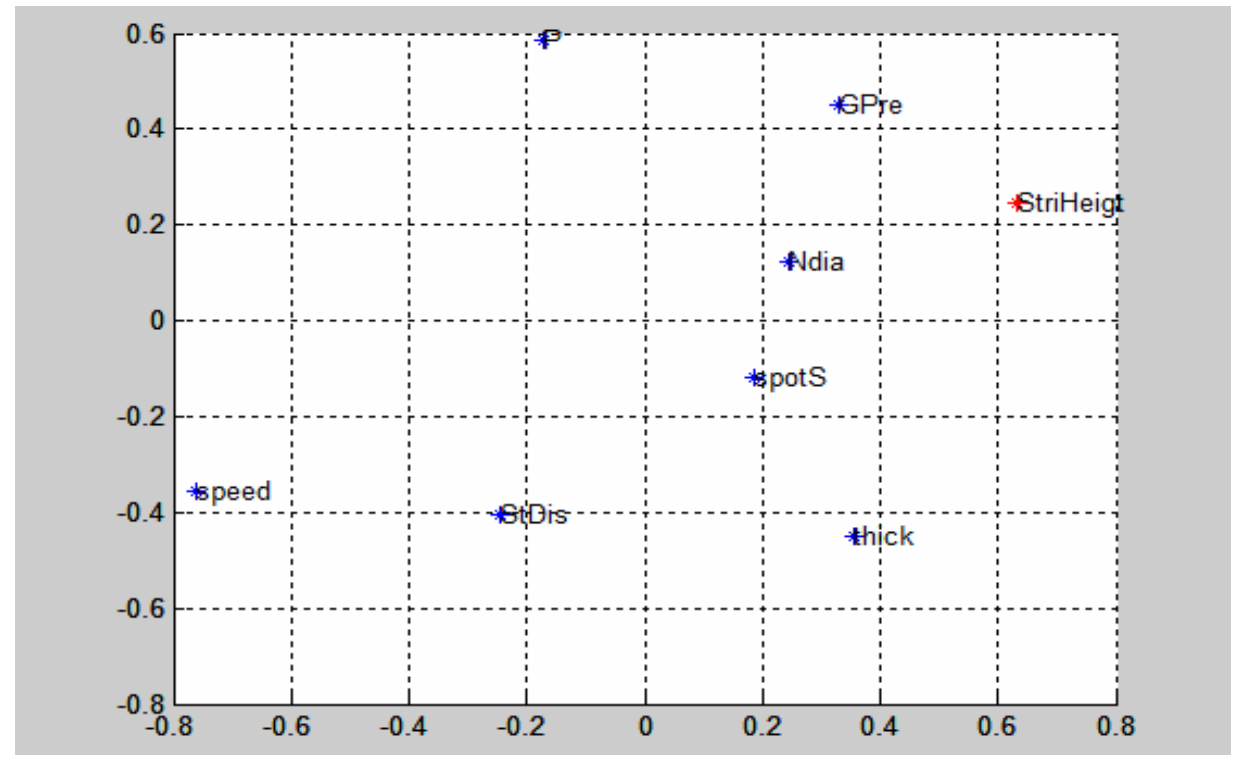

Figure 10: PLS loading plot.

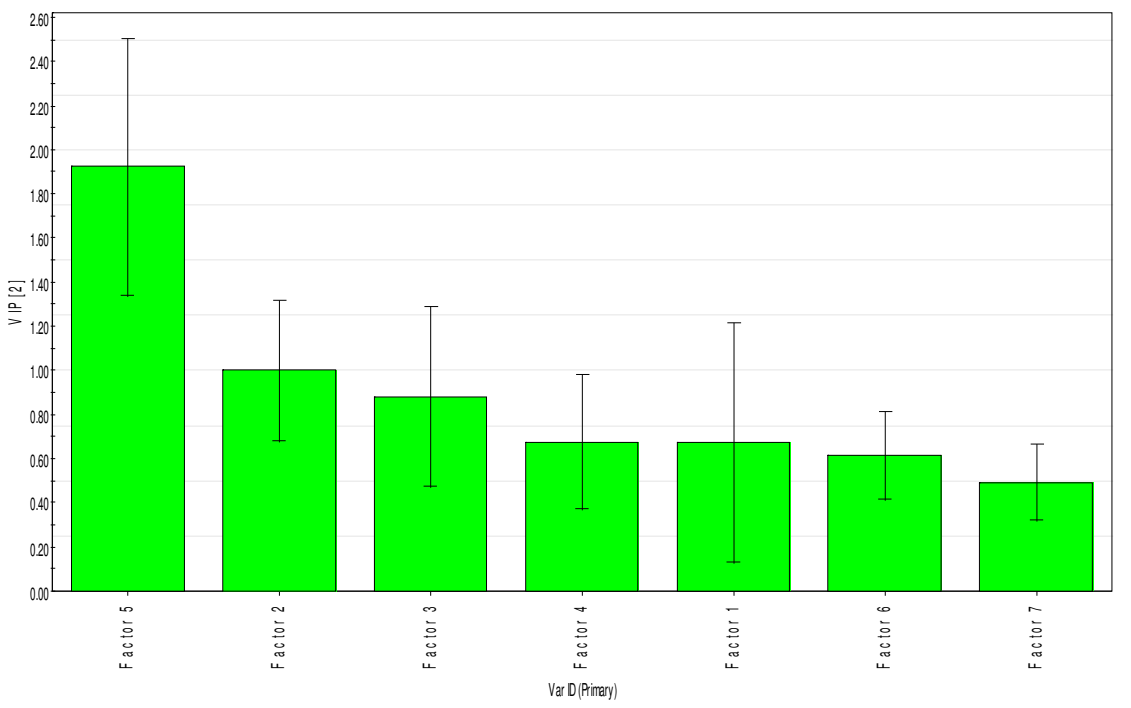

Figure 11: PLS VIP plot. 